Published in final edited form as:

Ann Vasc Surg. 2013 February ; 27(2): 186-193. doi:10.1016/j.avsg.2012.03.010.

\title{
Natural history of carotid artery occlusion
}

\author{
Darren Bryan, BS ${ }^{1}$, John Carson, MD $^{2}$, Heather Hall, MD $^{3}$, Qi He, MD $^{3}$, Khalil Qato, BA ${ }^{4}$, \\ Laurie Lozanski, $\mathbf{R V T}^{5}$, Susan McCormick, $\mathbf{P h D}^{3}$, and Christopher L. Skelly, $\mathbf{M D}^{3}$ \\ 1 University of Rochester, Rochester, NY, USA \\ ${ }^{2}$ University of California Davis, Sacramento, CA USA \\ ${ }^{3}$ University of Chicago, Department of Surgery, Chicago, IL, USA \\ ${ }^{4}$ New York Medical College, Valhalla, NY, USA \\ ${ }^{5}$ University of Chicago Vascular Lab, Chicago, IL, USA
}

\section{Abstract}

Carotid artery occlusion is a risk factor for stroke ipsilateral to the occlusion and puts patients in a high risk category when contralateral endarterectomy is performed. The purpose of this study was to evaluate the long-term outcomes of patients with internal carotid artery occlusion and to determine risk factors predictive of subsequent neurological event, contralateral carotid intervention, or death. Patients with internal carotid artery occlusion shown by duplex ultrasound were retrospectively identified and followed between January 2002 and June 2010 (follow-up 1 to 101 months, mean 52 months) at a tertiary care hospital. All had multiple duplex exams available for review. Chi-square analysis was used to determine risk factors for neurologic event, contralateral intervention, or all cause morality. Multivariate Cox proportional hazard analysis was conducted using univariate risk factors with $\mathrm{P}$ values $(<.1)$. Survival was estimated using the Kaplan-Meier method ( $\mathrm{p}<.05$ significant). 80 patients with internal carotid artery occlusion were identified and available for analysis. On initial encounter, 30(38\%) were symptomatic with $26(87 \%)$ having symptoms referable to the side of the occluded internal carotid artery. During follow-up, $7(9 \%)$ had a neurologic event, of which $6(86 \%)$ were referable to the occluded side; $14(18 \%)$ patients underwent a contralateral operation. 19(24\%) patients died during the period of study. Although numerous variables of multi-vessel disease were significant with Chi-square analysis, there was no significant risk factor associated with neurologic event upon multivariate analysis. The development of a hemodynamically significant stenosis $(>50 \%)$ or occlusion of the external carotid artery (ECA) ipsilateral to the occlusion on follow-up ( $\mathrm{p}<.027)$ was however, associated with increased risk of death. Kaplan-Meier analysis showed 7-year survival for patients with ECA disease at follow-up was significantly worse $(16.2 \% \pm 10.3 \%(\mathrm{n}=21)$ vs. $79 \% \pm 8.7 \%$ $(n=59) ; p<.00001)$. Frequently, patients present with neurological symptoms referable to the side of internal carotid artery occlusion. Eighty-six percent of neurologic events that occur in follow-up are attributable to the side of the occluded carotid, indicating that the occluded side continues to contribute to neurologic morbidity over time. Multivariate analysis revealed no single factor to be predictive of subsequent neurologic events. With significant risk of death in patients found to have

(C) 2012 Annals of Vascular Surgery Inc. Published by Elsevier Inc. All rights reserved.

Correspondence: Christopher L. Skelly, MD, University of Chicago Medical Center, 5841 S. Maryland Ave, MC 5028, Chicago, IL 60637, Phone: 773-702-6128, Fax: 773-702-0863, cskelly@surgery.bsd.uchicago.edu.

Society for Clinical Vascular Surgery Annual Symposium, March 16 ${ }^{\text {th }} 2011$, Orlando, FL

Publisher's Disclaimer: This is a PDF file of an unedited manuscript that has been accepted for publication. As a service to our customers we are providing this early version of the manuscript. The manuscript will undergo copyediting, typesetting, and review of the resulting proof before it is published in its final citable form. Please note that during the production process errors may be discovered which could affect the content, and all legal disclaimers that apply to the journal pertain. 
ipsilateral ECA stenosis during follow-up, it seems reasonable to continue surveillance of the occluded carotid.

\section{INTRODUCTION}

Carotid artery occlusion (CAO) in patients with atherosclerosis is a condition that adds to the complexity of cerebrovascular disease management, and is a known risk factor for stroke. Population studies offer estimates of the prevalence of symptomatic CAO to be $6 / 100,000$, while the true prevalence is assumed to be greater, ${ }^{1}$ particularly when considering those with transient symptoms who forgo evaluation. Additionally, the disease process has been recognized to be quite dangerous. It has been suggested that up to $15 \%$ of large artery infarctions may occur secondary to CAO, and that $27-38 \%$ of patients first present with significant stroke. ${ }^{1-2}$ After diagnosis, it has been reported that patients with CAO have a continued 2-5.5\% annual risk of stroke ipsilateral to the occlusion. ${ }^{3}$ In a study of patients with known internal carotid occlusion and contralateral asymptomatic carotid stenosis, AbuRahma found the combined risk of late stroke and TIA to be $10 \%$ per year ${ }^{4}$. Other studies have quoted the annual risk of stroke to be up to $6 \%$ after occlusion has occurred. ${ }^{5-8}$ Furthermore, $\mathrm{CAO}$ places patients who are undergoing contralateral carotid endarterectomy in a high-risk category, ${ }^{9}$ with perioperative risk of stroke or death reported to be $1.8-3.7 \% .{ }^{10-11}$ Perioperative risk of stroke or death in patients with known CAO undergoing contralateral carotid artery stenting is similarly high, with ranges between 3.3 and $7 \% .{ }^{12-13}$ Cumulative survival after diagnosis of internal carotid occlusion is poor, with 5 -year mortality due to all causes being $30-40 \% .5,14$

Few studies have been performed looking solely at outcomes and prognostic indicators in patients with unilateral carotid occlusion. Klijn found that previous ipsilateral TIA was associated with increased risk of recurrent stroke, ${ }^{15}$ and Paciaroni found age can predict mortality within 30 days of stroke. ${ }^{16}$ These studies, along with others, largely targeted patients after stroke occurrence. Given the nature of cerebrovascular disease, it would be beneficial to know the long term outcomes of patients with CAO in order to risk stratify patients to specific therapeutic modalities. Risk factors predictive of specific outcomes in patients with CAO have thus far gone largely undetected. With this study we aim to provide a contemporary natural history of patients with ICA occlusion, focusing on risk factors predictive of neurological event, intervention contralateral to occlusion, and death.

\section{METHODS}

All patients with complete internal carotid artery occlusion between 2002 and 2010 at a tertiary care hospital were identified as part of an IRB approved protocol. Of the patients identified, 80 had multiple ( 2 or more) duplex ultrasounds in follow-up and were selected for the study. Patient demographics, clinical presentation, radiology records, operative notes, and vascular lab reports were obtained and available for review. All comorbidities were documented from history and physical examination and the electronic medical record. Patients with only single Doppler ultrasounds or incomplete presenting data were excluded. Diagnosis of internal carotid artery occlusion was made with duplex ultrasound imaging.

All carotid duplex scans were performed with ATL HDI 3000 (Advanced Technology Laboratories, Bothel, WA), ATL HDI 5000, or Acuson Sequoia 512 (Acuson Corp, Mountain View, CA) ultrasound scanners with linear array 4-7 MHz or 5-10 MHz transducers. All DUS carotid studies were performed in our IAC accredited vascular laboratory (formerly ICAVL) by registered vascular technologists and were subsequently reviewed by board-certified vascular surgeons. The carotid arteries were examined with the patient in the supine position with the head slightly elevated and turned towards the 
contralateral side. The standard protocol for carotid duplex examination included examination of the CCA and the extra cranial segments of ICA, external carotid artery (ECA), and vertebral (VA) artery using spectral analysis and B-mode imaging complemented with color flow mapping. All velocity and B-mode data were recorded in the patient's vascular laboratory chart with representative images of velocity measurements and B-mode images stored as part of the electronic medical record.

The degree of stenosis contralateral to the occlusion was classified as previously described. ${ }^{17}$ A peak systolic velocity of $>155 \mathrm{~cm} / \mathrm{sec}$, an ICA/CCA ratio $\geq 2.0$ and enddiastolic velocity $<140 \mathrm{~cm} / \mathrm{sec}$ was used to diagnose a 50-79\% diameter stenosis. An enddiastolic velocity of $>140 \mathrm{~cm} / \mathrm{sec}$, along with a PSV of $\geq 370 \mathrm{~cm} / \mathrm{sec}$ and an ICA/CCA ratio of $\geq 6.0$ was used as the criteria for an $80-99 \%$ diameter-reducing stenosis. Other signs of disease, e.g., post-stenotic turbulence and visible plaque by B-mode image were also documented as supporting evidence of significant disease. ECA stenosis $>50 \%$ was defined as being present if peak systolic velocities greater than $200 \mathrm{~cm} / \mathrm{sec}$ coupled with evidence of post-stenotic turbulence and luminal reduction by B-mode image were detected in the ECA. ECA occlusion was defined as no evidence of detectable Doppler signal or color flow throughout the length of the ECA.

Patients were categorized according to initial presenting characteristics; as neurologically symptomatic (TIA, CVA, or amaurosis fugax) or asymptomatic. Presenting symptoms were additionally classified as referable to the side of the occlusion (non-referable to stenosis), or on the side contralateral to the occlusion (referable to stenosis). Study initiation occurred upon the date of first carotid ultrasound, and end points were defined as the onset of neurological complication, intervention contralateral to the occlusion, or all cause mortality. Stroke was defined as any localized neurologic deficit lasting $>24$ hours and confirmed by computed tomography (CT) scan or by magnetic resonance imaging, while TIA was defined as any neurologic deficit lasting $<24$ hours. Strokes were further categorized as major if the patient's activities of daily living were significantly impacted. Amaurosis fugax, TIA, and stroke were classified as referable to the side of the occlusion, or to the side contralateral to the occlusion. Death was determined primarily by hospital records and secondarily using the U.S. Social Security Death Index in patients who had no recent (within six months) available medical records verifying status. Doppler reports were additionally analyzed for progression of stenosis by advancement of category.

\section{Statistical Analysis}

Chi-square analysis was used to determine risk factors for neurologic event, contralateral intervention, or death. Those with $\mathrm{p}$-values $<.1$ were put in a multivariate Cox proportional hazard analysis. P-values $<.05$ were considered significant. Survival was estimated using the Kaplan-Meier method.

\section{RESULTS}

\section{Patient characteristics}

Of 201 patients initially identified with unilateral internal carotid artery occlusion, 81 had multiple duplex ultrasounds and complete presenting clinical information, and were entered in this study. One was excluded due to the presence of bilateral occlusion. Clinically relevant risk factors and group demographics are shown in table I. Mean follow-up time was 52 months with a range of 1 to 101 months.

On initial encounter, 30 patients (38\%) were symptomatic with 26 (87\%) having symptoms referable to the occluded carotid. Of the 26 patients with symptoms referable to the occluded carotid artery at the time of initial duplex demonstrating the occlusion, 10 patients had TIAs, 
7 patients had a major stroke and 9 patients had a minor stroke (table II). Two patients had multiple presenting symptoms; 1 with both TIA and amaurosis fugax, while the other had a major CVA with amaurosis fugax.

\section{Clinical outcomes}

During the follow-up period, 7(8.8\%) patients had a neurological event. Of these, 6(86\%) were hemispheric in nature and referable to the side of the occluded carotid artery. Over the eight-year period, this correlated with a $0.9 \%$ risk of neurological event referable to the occluded carotid per year. The events included 1 patient with amaurosis fugax, 2 with TIAs, and 3 with strokes. The sole neurological event referable to the non-occluded artery was a stroke (table III).

Overall survival for the entire cohort was $50 \pm 8 \%$ over the eight year time period (figure I), which corresponds to an annualized risk of death of $6.25 \% .19$ patients died during the time period of the study with the mean and median survival of 51 months. Five deaths (26\%) were determined to be cardiovascular in nature and five deaths (26\%) were due to cancer. No direct cause could be determined from available records for the remaining patients.

With respect to the outcome of the contralateral carotid artery, the majority of patients stayed within the same disease category on duplex ultrasound while 20(24\%) patients progressed in disease category. The majority of patients with progression went from $0-49 \%$ stenosis to $50-79 \%$ (18 patients, $23 \%$ ) and 2 (2.5\%) progressed from 50-79\% stenosis to $80-99 \%$ stenosis. There were three regressions, two that went from $80-99 \%$ to $50-79 \%$ $(2.5 \%)$, and one that went from $50-79 \%$ to $0-49 \%(1.3 \%)$. The average time from initial Doppler ultrasound to disease progression was 590 days (table III). 15(18.8\%) patients underwent a revascularization secondary to symptomatic lesions (7 patients, $9 \%$ ), progression of stenosis (5 patients, $6 \%$ ), or high-grade (80-99\%) lesions (3 patients, $4 \%$ ). The average time from initial Doppler ultrasound to intervention was 463 days. 9(11\%) patients underwent CEA and 5(6\%) patients had carotid artery stenting with embolic protection. The choice of the operative approach was left to the discretion of the operating surgeon. One patient underwent stenting of the ECA ipsilateral to the occlusion.

\section{Risk Factor analysis}

Chi-square analysis showed multiple variables to be significantly ( $\mathrm{p}<.1)$ associated with neurological event, contralateral intervention, or death (table IV). After multivariate analysis, no risk factor was found to be significantly $(\mathrm{p}<.05)$ associated with neurological event during follow-up. In particular, the presence or progression of atherosclerotic disease in other extracranial vessels did not predict subsequent neurologic event. Multivariate analysis additionally revealed that patients who had stenosis or occlusion of the external carotid ipsilateral to the occlusion during follow-up were at increased risk of death $(\mathrm{p}<.027)$. Kaplan-Meier analysis showed 8-year survival for patients with external carotid artery disease at follow-up to be significantly worse than for patients whose follow-up period was free of ECA disease $((16 \% \pm 10 \%(\mathrm{n}=21)$ vs. $69 \% \pm 8 \%(\mathrm{n}=59) ; \mathrm{p}<.00001))$ (figure II). A separate subgroup analysis was conducted on the $21(26 \%)$ patients with ipsilateral ECA stenosis or occlusion in follow up. Their relevant risk factors and demographics did not differ greatly from the rest of the study population, and are noted in Table V. One patient $(1.3 \%)$ had stenting of the external carotid artery secondary to the development of symptoms directly referable to the ECA distribution. Importantly, the annualized risk of death in the cohort of patients who had ECA stenosis or occlusion in follow up was $11 \%$ compared to those patients who did not have progression or stenosis, which was $4 \%$. 


\section{DISCUSSION}

In this study, the annualized risk of neurologic event during follow-up was found to be $1.1 \%$. Stroke rates have been quoted to drop from as high as $20 \%$ per annum at the time of occlusion, to $1.5-5 \%$ thereafter ${ }^{5,7}$ thereby making the initial occlusion the most dangerous time. In a prospective study of 212 patients in which carotid stenosis was followed to occlusion, Nicholls et al found 21 of $30(65 \%)$ strokes to occur in the hemisphere ipsilateral to the occlusion. ${ }^{5}$ Several other studies have shown similar conclusions, with strokes found to have a roughly $2 / 3$ predilection for the side of the occluded ICA. ${ }^{14}, 18-19$ This study confirms the finding that strokes trend toward the side of occlusion, albeit at a greater rate than previously reported, with $85 \%$ of events in follow-up referable to the occlusion.

Although this study was not designed to determine the cause of post-occlusion events, there are several mechanisms potentially contributing to stroke. First, transient hypotension can result in a low flow state and cause watershed infarction in poorly collateralized areas distal to occlusive lesions. Alternatively, embolism can occur via several mechanisms. The distal tail of the occluded ICA can fragment, showering the brain with emboli, or Stump Syndrome can occur. In this case, an occluded ICA has a proximally patent area, as well as a patent ECA showing retrograde flow. The ophthalmic artery, also with retrograde flow, serves as a collateral to the circulation of the middle cerebral artery. Embolism or low flow via this route is hypothesized to be a significant source of ipsilateral neurological symptomatology. ${ }^{20}$ Finally, trans-hemispheric migration through the Circle of Willis, while rare, can occur. In our study, all of the strokes ipsilateral to the CAO that occurred in followup were associated with low flow states secondary to hypotension with no evidence of stroke caused by stump syndrome. Compensatory hemodynamic mechanisms have been recognized in patients with unilateral occlusion of the internal carotid artery, and are particularly well studied in patients with asymptomatic presentations. Primarily, ICA occlusion is associated with subsequent increases in contralateral ICA flow. ${ }^{21-22}$ Insufficient increases in contralateral flow and poorly developed collateral vessels lead to neurologic symptomatology, particularly in low flow states. ${ }^{23}$ Other natural collaterals, namely the external carotid arteries ${ }^{24}$ and vertebral arteries ${ }^{25}$ play a role in compensation secondary to ICA occlusion. For this reason, we specifically looked at the ECA and Vertebral arteries in this study to look to see if disease of any of these collateral beds would predict neurologic event, contralateral operation or death. Only disease of the ECA in follow up was significant for predicting death. It may be that disease of the ECA in the cohort studied, may have contributed in some respect to neurologic events encountered in follow up. However, this did not reach significance and given the retrospective nature of the study, direct causality was not established.

The study was additionally designed to reveal factors prognostic of adverse neurologic events, intervention, and death. Upon multivariate analysis we found the development of occlusion or stenosis of the external carotid artery ipsilateral to the occluded internal carotid to be independently predictive of death $(\mathrm{p}<.0001)$. Kaplan-Meier analysis showed 8-year survival for this group to be significantly worse $(16 \% \pm 10.3 \%$ in the ECA occlusion cohort $(n=21)$ vs. $69 \% \pm 8 \%$ in patients without ECA occlusion $(n=59) ; p<.00001)$. Other studies following patients with $\mathrm{CAO}$ have found various clinical prognostic factors, including the size of initial infarcts, and advanced age to be associated with poor functional outcome. ${ }^{16,26}$ In one prospective study of 66 patients who presented with first-time stroke associated with ICA occlusion, Huang found previous TIA and anemia to be predictors of death and recurrent stroke within 2 years. Additionally, the degree of stenosis of the ipsilateral or contralateral CCAs, and contralateral ICA had no impact on death or recurrence of stroke. ${ }^{18}$ Other authors have reported a similar lack of prognostic value of the extra cranial 
vessels. ${ }^{16,27}$ To the best of our knowledge, this is the first instance of the external carotid arteries being identified as a significant clinical prognostic factor.

The significance of this finding is that it may identify patients with progressive atherosclerosis. It is tempting to indemnify ECA occlusion as causative for events ipsilateral to the occlusion; however, in multivariable analysis, ipsilateral ECA progression of stenosis or occlusion was not significant for subsequent neurologic event. Several studies have considered the role and importance of the ECA in providing collateral flow. In a prospective analysis of the contribution of the ECA ipsilateral to internal carotid occlusion, van Laar found $20 \%$ of patients to have Grade 0 (no filling of ophthalmic artery) collateral ECA flow, $20 \%$ to have Grade 1 (filling of carotid siphon) collateral flow, and 60\% to have Grade 2 (filling of anterior/middle cerebral artery) flow. ${ }^{24}$ In a separate study, also by van Laar, flow territory maps in patients with CAO showed that the middle cerebral artery and anterior cerebral artery distributions were supplied mainly by the vertebrobasilar system and contralateral ICA respectively. ${ }^{28}$ With the ECA playing a variably important role during ICA occlusion, it stands to reason that its occlusion would not necessarily result in neurovascular symptomatology. This in part could explain why ECA occlusion was not found to be predictive of adverse neurological events, and supports it instead as a marker for progressive atherosclerosis. It is well recognized that atherosclerosis is a systemic process, and it is rare to have a singular area of disease. ${ }^{29}$ Various factors have been proposed to have an influence on the distribution of lesions. These include modifiable risk factors, as well as a complex array of genetic variants influencing hemodynamics, activity of lipoprotein oxidation pathways, and immune status. ${ }^{30}$ The interplay between several vascular beds, including the femoral, carotid, and coronary distributions, has been particularly well studied. Some of the strongest associations have been drawn between carotid and coronary disease, with common carotid artery intima-media thickness correlating with clinical coronary events..$^{31-32}$ One group found each $0.03-\mathrm{mm}$ increase in intima-media thickness per year to be associated with a relative risk of 2.2 for non-fatal MI or coronary death. ${ }^{33}$ Protocols for measuring intima-media thickness vary, but those which combine views of the common carotid, carotid bifurcation, and internal carotid have been found to be the most precise and reproducible. ${ }^{34,35}$ Intima-media thickness of the external carotid artery has not been sufficiently studied, and its prognostic value is unknown.

Our study had several limitations. First, we were only able to verify the cardiovascular causes of death of 5 of the 19 patients who died. By utilizing the U.S. Social Security Death Index, we minimized the risk of artificially recording patients who died outside the hospital system as living. However, the Index includes only dates and no information on cause of death. This information would be valuable, particularly in establishing a potential link between the development of external carotid disease and death due to cardiovascular complication.

Secondly, because screening for carotid stenosis and occlusion is frequently not performed until symptoms become clinically apparent, it is difficult to establish a time line for the progression of stenosis and subsequent vessel occlusion. While we attempted to begin our survey of each patient as soon as their occluded carotid artery became evident, the early stages of the disease course could have been missed. This could lead to an overestimation of the rate of stenosis of the vessel contralateral to the occlusion, however determining this rate was not the primary goal of the study. Additionally, some patients had undergone endarterectomy on the ICA contralateral to the occlusion prior to study initiation. Restenosis after endarterectomy has been reported to occur at a slower rate than in a native vessel ${ }^{36}$, therefore this could potentially lengthen the time to progression/intervention. 
By excluding patients with only a single ultrasound, a large amount of demographic data was lost. However, many excluded patients also had incomplete presentation data available in their electronic medical records. In many cases this was due to only a brief duration of care at our center.

Finally, by including only a patient population that has multiple duplex ultrasounds, a selection bias was created towards those with ongoing vascular disease. We did, however, observe only $38 \%$ of patients to present symptomatically, while some have found that up to $72 \%$ of patients with internal carotid occlusion present symptomatically. ${ }^{14}$ It is well recognized that presenting symptomatology in patients with CAO has substantial effects on prognosis. ${ }^{23}$ As previously mentioned, those who are initially asymptomatic tend to have more effective collateralization, and subsequently experience fewer recurrent symptoms. Furthermore, the cohort of patients with follow-up significant for ipsilateral ECA stenosis or occlusion presented in a relatively benign manner, with only $22 \%$ being symptomatic.

\section{CONCLUSION}

Patients with carotid occlusion frequently present with symptoms referable to the occlusion. $86 \%$ of neurologic events were ipsilateral to the occluded internal carotid artery. This correlated with an annual risk of neurologic event of $1.1 \%$. Multivariate analysis confirmed a significant risk of death in patients found to have ipsilateral ECA stenosis or occlusion during follow-up. Annualized mortality was $11 \%$ in this cohort of patients suggesting that aggressive surveillance and monitoring may be warranted in this group. Additionally, future study of the utility of the external carotid artery in prognostication of cardiovascular events is warranted.

As we further our understanding of the natural history of carotid disease in patients with CAO, we may be better able to predict those patients at risk for a neurologic event and potentially alter our disease management to lower subsequent events in these patients.

\section{Acknowledgments}

Dr. Skelly is funded by NIH K-08-HL091053 as well by the American Vascular Association/American College of Surgeons and NHLBI Jointly Sponsored Mentored Clinical Scientist Development Award.

The contents are solely the responsibility of the authors and do not necessarily represent the official views of the NHLBI or the NIH

\section{References}

1. Flaherty M, Flemming K, McClelland R, et al. Population-based study of symptomatic internal carotid artery occlusion: incidence and long-term follow-up. Stroke. 2004; 35(8):e349-e52. [PubMed: 15232124]

2. Fritz V, Voll C, Levien L. Internal carotid artery occlusion: clinical and therapeutic implications. Stroke. 1985; 16(6):940-4. [PubMed: 4089925]

3. Klijn C, Kappelle L, Tulleken C, et al. Symptomatic carotid artery occlusion. A reappraisal of hemodynamic factors. Stroke. 1997; 28(10):2084-93. [PubMed: 9341723]

4. AbuRahma A, Stone P, Abu-Halimah S, et al. Natural history of carotid artery occlusion contralateral to carotid endarterectomy. J Vasc Surg. 2006; 44:62-6. [PubMed: 16828427]

5. Nicholls S, Kohler T, Bergelin R, et al. Carotid artery occlusion. J Vasc Surg. 1986; 4:479-485. [PubMed: 3773131]

6. Barnett H, Peerless S, Kaufmann J. "Stump" on internal carotid artery--a source for further cerebral embolic ischemia. Stroke. 1978; 9(5):448-56. [PubMed: 705825] 
7. Cote R, Barnett H, Taylor D. Internal carotid occlusion: a prospective study. Stroke. 1983; 14(6): 989-02.

8. Norris J, Zhu C, Bornstein N, et al. Vascular risks of asymptomatic carotid stenosis. Stroke. 1991; 22:1485-90. [PubMed: 1962321]

9. Ferguson G, Eliasziw M, Barr H, et al. The North American Symptomatic Carotid Endarterectomy Trial : surgical results in 1415 patients. Stroke. 1999; 30(9):1751-8. [PubMed: 10471419]

10. Rockman C, Su W, Lamparello P, et al. A reassessment of carotid endarterectomy in the face of contralateral carotid occlusion: surgical results in symptomatic and asymptomatic patients. J Vasc Surg. 2002; 36(4):668-73. [PubMed: 12368723]

11. Maatz W, Köhler J, Botsios S, et al. Risk of stroke for carotid endarterectomy patients with contralateral carotid occlusion. Ann Vasc Surg. 2008; 22(1):45-51. [PubMed: 18083336]

12. Sabeti S, Schillinger M, Mlekusch W, et al. Contralateral high-grade carotid artery stenosis or occlusion is not associated with increased risk for poor neurologic outcome after elective carotid stent placement. Radiology. 2004; 230(1):70-6. [PubMed: 14695388]

13. Touzé E, Trinquart L, Chatellier G, et al. Systematic review of the perioperative risks of stroke or death after carotid angioplasty and stenting. Stroke. 2009; 40(12):e683-e93. [PubMed: 19892997]

14. Faught W. Presentation and natural history of internal carotid artery occlusion. J Vasc Surg. 1993; 18(3):512-23. [PubMed: 8377246]

15. Klijn C, Kappelle L, van Huffelen A, et al. Recurrent ischemia in symptomatic carotid occlusion: Prognostic value of hemodynamic factors. Neurology. 2000; 55:1806-12. [PubMed: 11134377]

16. Paciaroni M, Caso V, Venti M, et al. Outcome in patients with stroke associated with internal carotid artery occlusion. Cerebrovasc Dis. 2005; 20:108-13. [PubMed: 16006758]

17. Shaalan W, Wahlgren C, Desai T, et al. Reappraisal of velocity criteria for carotid bulb/internal carotid artery stenosis utilizing high-resolution B-mode ultrasound validated with computed tomography angiography. J Vasc Surg. 2008; 48(1):104-12. [PubMed: 18486416]

18. Hankey G, Warlow C. Prognosis of symptomatic carotid artery occlusion. Cerebrovasc Dis. 1991; $1: 245-56$.

19. Fields W, Lemak N. Joint study of extracranial arterial occlusion. X. Internal carotid artery occlusion. JAMA. 1976; 235:2734-8. [PubMed: 946886]

20. Mohr, J.; Gautier, J.; Pessin, M. Internal carotid artery disease. Barnett, HJM.; M, J.; Stein, BM.; Yatsu, FM., editors. Philadelphia, Pa: WB Saunders Co; 1998.

21. AbuRahma A, Richmond B, Robinson P, et al. Effect of contralateral severe stenosis or carotid occlusion on duplex criteria of ipsilateral stenoses: comparative study of various duplex parameters. J Vasc Surg. 1995; 22(6):751-61. [PubMed: 8523610]

22. Alexander J, Moawad J, Super D. Outcome analysis of carotid artery occlusion. Vasc Endovascular Surg. 2007; 41(5):409-16. [PubMed: 17942856]

23. Power W, Derdeyn C, Fritsch S, et al. Benign prognosis of never-symptomatic carotid occlusions. Neurology. 2000; 54:878-82. [PubMed: 10690980]

24. van Laar P, van der Grond J, Bremmer J, et al. Assessment of the contribution of the external carotid artery to brain perfusion in patients with internal carotid artery occlusion. Stroke. 2008; 39(11):3003-8. [PubMed: 18688004]

25. Nicolau C, Gilabert R, García A, et al. Effect of internal carotid artery occlusion on vertebral artery blood flow: a duplex ultrasonographic evaluation. J Ultrasound Med. 2001; 20(2):105-11. [PubMed: 11211130]

26. Huang W, Weng W, Chien Y, et al. Predictive factors of outcome and stroke recurrence in patients with unilateral atherosclerosis-related internal carotid artery occlusion. Neurol India. 2008; 56:173-8. [PubMed: 18688143]

27. Verlato F, Camporese G, Bernardi E, et al. Clinical outcome of patients with internal carotid artery occlusion: A prospective follow-up study. J Vasc Surg. 2000; 32:293-8. [PubMed: 10917989]

28. van Laar P, Hendrikse J, Klijn C, et al. Symptomatic carotid artery occlusion: flow territories of major brain-feeding arteries. Radiology. 2007; 242(2):526-34. [PubMed: 17255422]

29. Rothwell P. The interrelation between carotid, femoral and coronary artery disease. Eur Heart J. 2001; 22(1):11-4. editorial. [PubMed: 11133203] 
30. VanderLaan P, Reardon C, Gets G. Site specificity of atherosclerosis siteselective response to atherosclerotic modulators. ATVB. 2004; 24:12-22.

31. Touboul P-J, Vicaut E, Labreuche J, et al. Correlation between the Framingham risk score and intima media thickness: The Paroi Artérielle et Risque Cardio-vasculaire (PARC) study. Atherosclerosis. 2007; 192(2):363-9. [PubMed: 16769075]

32. O'Leary DH, Polak JF, Kronmal RA, et al. Carotid-artery intima and media thickness as a risk factor for myocardial infarction and stroke in older adults. Cardiovascular Health Study Collaborative Research Group. N Engl J Med. 1999; 340:14-22. [PubMed: 9878640]

33. Hodis H, Mack W, LaBree L, et al. The role of carotid artery intima-media thickness in predicting clinical coronary events. Ann Int Med. 1998; 128(4):262-9. [PubMed: 9471928]

34. Dogan S, Duivenoorden R, Grobbee D, et al. Ultrasound protocols to measure carotid intimamedia thickness in trials; comparison of reproducibility, rate of progression, and effect of intervention in subjects with familial hypercholesterolemia and subjects with mixed dyslipidemia. Ann Med. 2010; 42(6):447-64. [PubMed: 20645885]

35. Dogan S, Plantinga Y, Evans GW, et al. OPAL investigators. Ultrasound protocols to measure carotid intima-media thickness: a post-hoc analysis of the OPAL study. Curr Med Res Opin. 2009; 25:109-22. [PubMed: 19210144]

36. Raman K, Layne S, Makaroun M, et al. Disease progression in contralateral carotid artery is common after endarterectomy. J Vasc Surg. 2004; 39(1):52-7. [PubMed: 14718813] 


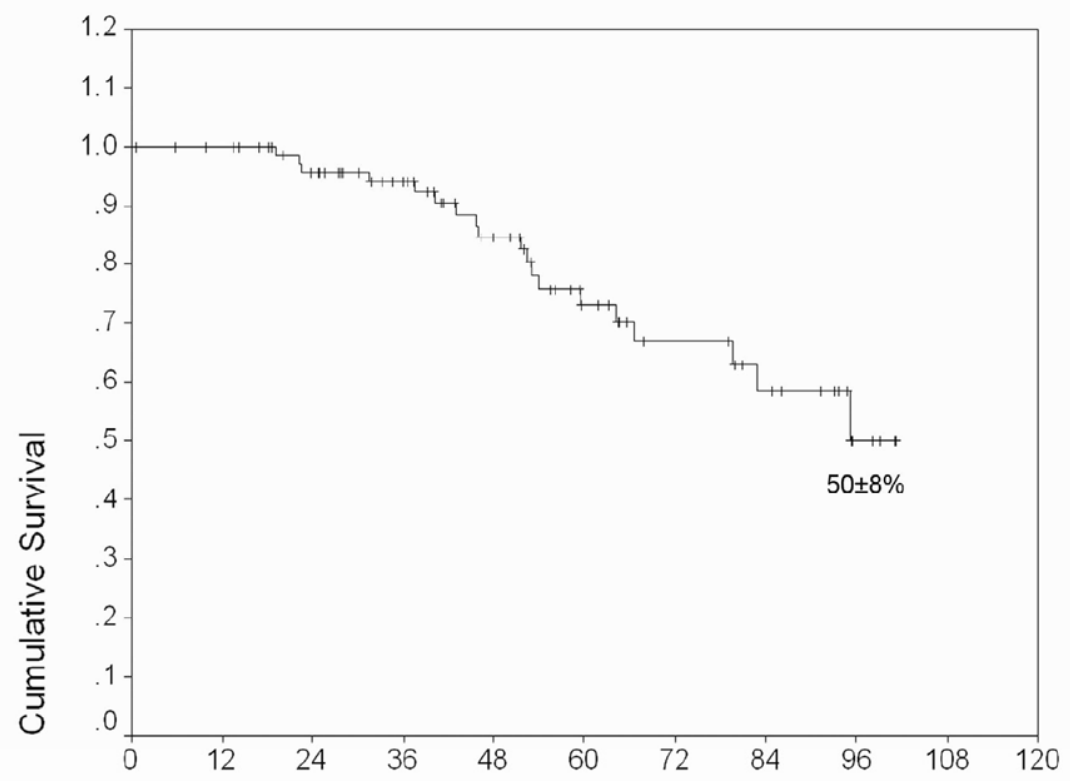

Number at risk

80

Time (Months)

Figure 1.

Cumulative 8 year survival of a cohort of 80 patients with occluded Internal Carotid artery on duplex ultrasound. 


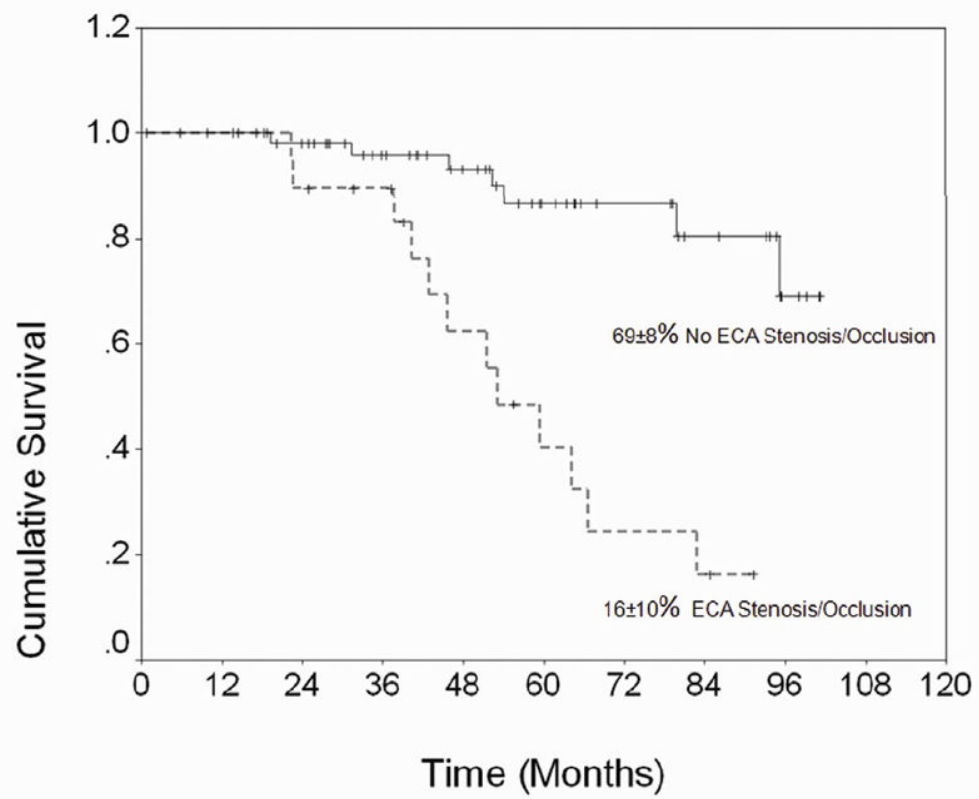

$\begin{array}{lrrrrrrrrrr}\text { No ECA Stenosis/Occlusion } & 59 & 57 & 50 & 40 & 32 & 22 & 16 & 10 & 4 & 0 \\ \text { ECAStenosis/Occlusion } & 21 & 20 & 17 & 15 & 9 & 5 & 3 & 2 & 1 & 0\end{array}$

Figure 2.

Kaplan-Meier analysis of the 8-year survival of patients with ECA stenosis or occlusion in follow-up $(\mathrm{n}=21)$ compared to patients who had no stenosis or occlusion in follow up $(16 \pm 10 \%$ vs. $69 \pm 8 \% ; \mathrm{p}<0.00001)$. 


\section{Table I}

Demographics and Risk factors of patients on initial presentation.

\begin{tabular}{ll}
\hline Risk/Demographic Factor & (n=80) \\
\hline Average age & 65 \\
Male & $40(50 \%)$ \\
African-American & $41(51 \%)$ \\
Smoking at diagnosis & $25(31 \%)$ \\
History of smoking & $68(84 \%)$ \\
Hypertension & $69(86 \%)$ \\
Hyperlipidemia & $51(65 \%)$ \\
History of CVA or TIA & $27(35 \%)$ \\
Insulin Dependent Diabetes Mellitus & $8(10 \%)$ \\
Non-Insulin Dependent Diabetes Mellitus & $12(15 \%)$ \\
Chronic Kidney Disease & $8(10 \%)$ \\
End Stage Renal Disease & $5(6 \%)$ \\
Previous intervention on contralateral Carotid & $17(21 \%)$ \\
Coronary Artery Disease & $34(43 \%)$ \\
Symptomatic at presentation & $30(38 \%)$ \\
\hline
\end{tabular}

$T I A$, Transient Ischemic Attack; $C V A$, Stroke. 


\section{Table II}

Localization and frequency of presenting symptoms;

\begin{tabular}{ll}
\hline Presenting Symptom & $(\mathbf{n = 3 0 )}$ \\
\hline Referable to Occlusion & $\mathbf{2 6}(\mathbf{8 7 \%})$ \\
Referable to Contralateral Carotid & $\mathbf{4 ( 1 3 \% )}$ \\
Amurosis Fugax - referable to contralateral carotid & $3(10 \%)$ \\
Amurosis Fugax - referable to occlusion & 0 \\
TIA - referable to contralateral carotid & $2(7 \%)$ \\
TIA - referable to occlusion & $10(33 \%)$ \\
CVA (major)- referable to contralateral carotid & 0 \\
CVA (major)- referable to occlusion & $7(23 \%)$ \\
CVA (minor) - referable to contralateral carotid & $2(7 \%)$ \\
CVA (minor) - referable to occlusion & $9(30 \%)$ \\
\hline
\end{tabular}

TIA, Transient Ischemic Attack; $C V A$, Stroke. 
Table III

Analysis of endpoints reached during the study time.

\begin{tabular}{ll}
\hline Endpoints Reached in Follow-up & (n=80) \\
\hline Total with Neurologic Symptoms & $7(8.8 \%)$ \\
\hline Neurologic Symptoms Referable to Occlusion & $6(7.5 \%)$ \\
•Amurosis Fugax & $1(1.3 \%)$ \\
•TIA & $2(2.5 \%)$ \\
$\bullet$ CVA & $3(3.8 \%)$ \\
\hline Neurologic Symptoms Referable to Contralateral Carotid & $1(1.3 \%)$ \\
•Amurosis Fugax & 0 \\
•TIA & 0 \\
$\bullet$ CVA & $1(1.3 \%)$ \\
\hline Contralateral Revascularization & $15(18.8 \%)$ \\
Average Time to Revascularization & $463 \mathrm{Days}$ \\
Death & $19(24 \%)$ \\
\hline
\end{tabular}

$T I A$, Transient Ischemic Attack; $C V A$, Stroke. 


\section{Table IV}

$\mathrm{P}$ values from Chi squared analysis of risk factors predictive of contralateral operation, neurologic event in follow-up, and death.

\begin{tabular}{llll}
\hline Risk Factor & Contralateral operation & Neurologic Event in follow up & Death \\
\hline Prior Neck irradiation & 0.68 & 0.17 & $.054^{\mathcal{S}}$ \\
Symptoms present at initial presentation & 0.57 & 0.47 & 0.075 \\
Amurosis Fugax at initial presentation & $0.078^{\mathcal{S}}$ & 0.76 & 0.44 \\
Contralateral ICA progression in follow up & 0.20 & 0.47 & 0.49 \\
Ipsilateral ECA at presentation & 0.17 & 0.19 & 0.005 \\
Contralateral ECA at presentation & 0.005 & 0.56 & 0.014 \\
Ipsilateral Vert in follow up & & 0.019 & 0.29 \\
Ipsilateral ECA in follow up & 0.033 & 0.073 & $<.0001^{*}$ \\
Contralateral ECA in follow up & 0.62 & 0.46 & 0.061 \\
Any ECA/Vertebral stenosis or occlusion at presentation & 0.053 & 0.098 & 0.007 \\
ECA/Vertebral stenosis or occlusion progression in follow up & 0.29 & 0.427 & 0.31 \\
\hline
\end{tabular}

p $<.05$ in multivariate analysis.

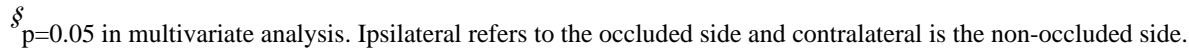

ICA, Internal Carotid Artery; ECA, External Carotid Artery; Vert, Vertebral artery. 


\section{Table V}

Demographics of patients with ipsilateral ECA stenosis or occlusion in follow up.

\begin{tabular}{ll}
\hline Risk Factor & $(\mathbf{n = 2 1})$ \\
\hline Symptomatic at Presentation & $4(22 \%)$ \\
Smoking History & $15(83 \%)$ \\
Hypertension & $15(83 \%)$ \\
Hyperlipidemia & $11(61 \%)$ \\
NIDDM & $2(11 \%)$ \\
CAD & $3(17 \%)$ \\
Chronic Kidney Disease & $2(11 \%)$ \\
End Stage Renal Disease & $1(5 \%)$ \\
Male & $9(50 \%)$ \\
ECA Intervention & $1(5 \%)$ \\
\hline
\end{tabular}

$N I D D M$, type 2 Non-insulin dependent Diabetes mellitus; $C A D$, Coronary artery disease; $E C A$, External Carotid Artery. 